\title{
Chern-Simons Particles with Nonstandard Gravitational Interaction
}

\author{
J. Lukierski \\ Institute for Theoretical Physics, University of Wrocław, \\ pl. Maxa Borna 9, 50-204 Wrocław, Poland \\ email: lukier@ift.uni.wroc.pl \\ and \\ Departamento de Fisica Teorica, Universidad de Valencia, \\ Av. dr Moliner 50, E-46100 Burjasot (Valencia), Spain \\ P.C. Stichel \\ An der Krebskuhle 21 \\ D-33619 Bielefeld, Germany \\ e-mail:pstichel@gmx.de \\ W.J. Zakrzewski
Department of Mathematical Sciences, University of Durham,
Durham DH1 3LE, UK
e-mail: W.J.Zakrzewski@durham.ac.uk
and
Center for Theoretical Physics
Massachusetts Institute of Technology, Cambridge, 02139, USA
}

\begin{abstract}
The model of nonrelativistic particles coupled to nonstandard (2+1)-gravity [1] is extended to include Abelian or non-Abelian charges coupled to ChernSimons gauge fields. Equivalently, the model may be viewed as describing the (Abelian or non-Abelian) anyonic dynamics of Chern-Simons particles coupled, in a reparametrization invariant way, to a translational Chern-Simons action. The quantum two-body problem is described by a nonstandard Schrödinger equation with a noninteger angular momentum depending on energy as well as particle charges. Some numerical results describing the modification of the energy levels by these charges in the confined regime are presented. The modification involves a shift as well as splitting of the levels.
\end{abstract}




\section{Introduction}

Particles in $2+1$ dimensions $(D=2+1)$ carrying electric (Abelian) or isospin (nonAbelian) internal charges coupled to Chern-Simons (CS) gauge fields, have been considered in many applications (see e.g. [2,3]). These particles, in the Abelian case describing anyons (see e.g. [4]) and for non-Abelian couplings their generalizations ("non-Abelian anyons"), are characterized, respectively, by the Abelian and nonAbelian versions of braided fractional statistics (see e.g. $[5,6]$ ).

The aim of this paper is to supplement the dynamics of nonrelativistic CS particles with nonstandard gravitational interactions described in [1]. The free field actions in our model for the $D=2+1$ gravitational and gauge fields are described by the CS Lagrangians. In particular, in the gravitational sector described by dreibeins $E_{\frac{a}{\mu}}$ $(\mu=0,1,2 ; \underline{a}=1,2)$, with tangent space indices restricted to nonrelativistic $S O(2)$ space rotations, we use the following action proposed in [1]:

$$
S_{0}^{\mathrm{GR}}=\frac{1}{2 \lambda} \int d^{3} x \epsilon^{\mu \nu \rho} E_{\frac{a}{\mu}} T_{\nu \rho}^{a}+S_{\mathrm{B}}
$$

where $T_{\mu \nu}^{a}=\partial_{\mu} E_{\nu}^{a}-\partial_{\nu} E \frac{a}{\mu}$ describes the $D=2+1$ torsion field and $S_{\mathrm{B}}$ are boundary terms specified in $[1,12]$. The dreibeins transform covariantly under local space translations (fixed time diffeomorphisms). Then the invariant free action for nonrelativistic point particles $\rrbracket$ described by trajectories $x_{\alpha}^{i}(t)(i=1,2 ; \alpha,=1 \ldots N)$ is given, in the first order formalism, by $[1,12]$

$$
S_{\mathrm{part}, 0}^{(\mathrm{N})}=\int d t \sum_{\alpha=1}^{N}\left(\xi \frac{\underline{a}}{\alpha}\left(E_{j, \alpha}^{\frac{a}{x_{\alpha}^{j}}}+E_{0, \alpha}^{\underline{a}}\right)-\frac{1}{2} \xi \frac{a}{\alpha} \xi \frac{a}{\alpha}\right) .
$$

In the gauge sector we consider the known free CS actions:

i) Abelian case $\left(A_{\mu}\right.$ - electromagnetic potential)

$$
S_{0}^{\mathrm{A}}=\frac{\kappa}{4} \int d^{3} x \epsilon^{\mu \nu \rho} A_{\mu} F_{\nu \rho}=\frac{\kappa}{2} \int d^{3} x \epsilon^{\mu \nu \rho} A_{\mu} \partial_{\nu} A_{\rho},
$$

where $F_{\mu \nu}=\partial_{\mu} A_{\nu}-\partial_{\nu} A_{\mu}$

ii) Non-Abelian case $\left(A_{\mu}^{i}\right.$ - isospin gauge field potential; for simplicity we shall choose the internal symmetry group $G=S U(2))$ (cp. [8]):

$$
S_{0}^{\mathrm{NA}}=\frac{\kappa}{2} \int d^{3} x \epsilon^{\mu \nu \rho}\left(A_{\mu}^{i} \partial_{\nu} A_{\rho}^{i}+\frac{1}{3} \epsilon_{i j k} A_{\mu}^{i} A_{\nu}^{j} A_{\rho}^{k}\right) .
$$

In the Abelian case the "charge space" is trivial, described by a constant numerical parameter. In the non-Abelian case the internal degrees of freedom of CS particles should be explicitly taken into consideration by extending the space-time geometry (see e.g. [9-10]). The non-Abelian charge space coordinates $Q^{i}(t)$ carrying the adjoint representation of internal symmetry group $G$ (in our case $i=1,2,3$ and $Q^{i}$ is the

\footnotetext{
${ }^{1}$ For reasons of simplicity we give all particles the same mass $m=1$ in appropriate units.
} 
$S O(3)$ isovector), after quantization $\left(Q^{i} \rightarrow \hat{Q}^{i}\right)$, constitute the quantum mechanical analog of current algebra coordinates, with Lie-algebraic equal time commutation relations

$$
\left[\hat{Q}^{i}(t), \hat{Q}^{j}(t)\right]=i \epsilon^{i j k} \hat{Q}^{k}(t) .
$$

It is convenient to put the coordinates $Q^{a}$ on the sphere $S^{2}$ of radius $J$ :

$$
Q^{i} Q^{i}=J^{2}
$$

which describes an adjoint symplectic orbit of $S U(2)$ with the following Kirillov symplectic two form $[9]$

$$
\Omega=\frac{1}{2 J^{2}} \epsilon^{i j k} Q^{i} d Q^{j} d Q^{k}
$$

After quantization the relation (1.6) defines the Casimir of $S U(2) \simeq S O(3)$ algebra (1.5) which implies the quantization of the radius $J$ by integers and half-integers. Using Darboux variables one can derive from (1.7) the free action for charge coordinates $Q^{i}$ (see Sect. 3). We would like to recall here that the dynamics of free particles on space-time $\times S^{2}$ manifold was first derived in the Kaluza-Klein framework [11] leading to $Q^{i}$ which satisfy the Wong equations [10].

This paper can be regarded as the extension of our results in $[1,12]$, where we have considered the interaction of $D=2$ nonrelativistic particles and of the gravitational

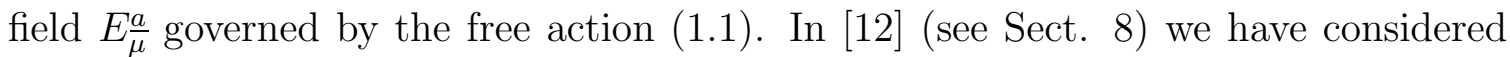
also the interaction with a constant $D=2$ magnetic field. In this paper we consider the additional dynamical Abelian and non-Abelian CS gauge fields. Interestingly enough the coupling to dynamical gauge fields appears simpler than the interaction with fixed external gauge potentials. This simplicity follows from the main property of the CS interactions in $D=2+1$, the local field - current identity, which permits us to solve algebraically the fields in terms of the sources (see e.g. $[7,8]$ ). In consequence, we obtain the solvability of the $D=2+1$ two-body problem with gravitational and CS gauge interactions. It should be mentioned here that an analogous problem for $D=1+1[13,14]$ can be also solved; however, the field-current identity looses its local character (see [15]).

The plan of our paper is as follows:

In Sect. 2 we extend the model given in [1] by including the coupling to the $D=2+1$ CS electrodynamics (see (1.3)) and present the classical dynamics.

In Sect. 3 we consider the extension of the notion of point particles in spacetime to non-Abelian CS particles ([8-11] with space-time points supplemented by internal charge coordinates $Q^{i}$, constrained by (1.6). The non-Abelian gauge sector is described by the CS action (1.4). We find that, after quantization, the energy levels of the two-body problem depend on the eigenvalues of the following isospinlike operator

$$
\frac{\hat{\Omega}}{2}:=\hat{Q}_{1}^{i} \hat{Q}_{2}^{i}=\frac{1}{2}\left(\hat{J}_{12}^{2}-\hat{J}_{1}^{2}-\hat{J}_{2}^{2}\right)
$$


where $\hat{J}_{12}^{2}=\left(\hat{Q}_{1}^{i}+\hat{Q}_{2}^{i}\right)^{2}, \hat{J}_{1}^{2}=\left(\hat{Q}_{1}^{i}\right)^{2}, \hat{J}_{2}^{2}=\left(\hat{Q}_{2}^{i}\right)^{2}$. Thus, if the eigenvalues of the individual particles are $j_{1}\left(j_{1}+1\right)$ and $j_{2}\left(j_{2}+1\right)$ respectively, the eigenvalues of $\hat{J}_{12}^{2}$, are given by $j_{12}\left(j_{12}+1\right)$ where $j_{12}$ lies between $\left|j_{1}+j_{2}\right|$ and $\left|j_{1}-j_{2}\right|$. In both cases of electric and isospin interactions, in Sect. 2 and Sect. 3 we present the modification of the classical results given in $[1,12]$ which did not include the gauge interactions. In Section 4 we describe two-body quantum mechanics and present numerical results for the corresponding modification of the energy spectra in the confined regime for both the Abelian and the non-Abelian case.

Sect. 5 presents some outlook.

\section{Classical Dynamics for the $D=2$ Abelian Case}

We consider the following action of $N$ nonrelativistic charged particles interacting with dreibein fields $E_{\mu}^{a}$ and an electromagnetic field $A_{\mu}$ :

$$
S_{\text {part }}^{(\mathrm{N})}=S_{\text {part }, 0}^{(\mathrm{N})}+\int d t \sum_{\alpha=1}^{N} e_{\alpha}\left(A_{j, \alpha} \dot{x}_{\alpha}^{j}+A_{0, \alpha}\right),
$$

where $e_{\alpha}$ is the electric charge of the $\alpha$-th particle. Under the assumption that the fields $A_{\mu}(\vec{x}, t)$ transform covariantly under fixed time diffeomorphism $S_{\text {part }}^{(\mathrm{N})}$ is an invariant entity.

The full action is given now by

$$
S^{(\mathrm{N})}=S_{0}^{\mathrm{GR}}+S_{0}^{\mathrm{A}}+S_{\mathrm{part}}^{(\mathrm{N})} .
$$

The equation of motion (EOM), the Gauss constraint for the dreibeins $E \frac{a}{\mu}$ derived from $(2.2)$ and their solution are described in $[1,12]$. We have

$$
E \frac{a}{\mu}(\vec{x}, t)=-\frac{\lambda}{4 \pi} \partial_{\mu} \sum_{\alpha} \xi \frac{a}{\alpha} \phi\left(\vec{x}-\vec{x}_{\alpha}\right)+E_{\mu}^{a s, \underline{a}},
$$

with

$$
\begin{gathered}
E_{i}^{a s, \underline{a}}=\delta_{i}^{a}, \\
E_{0}^{a s, \underline{a}}=-v^{\underline{a}}(t),
\end{gathered}
$$

where the singular gauge function $\phi$ is defined by

$$
\phi(\vec{x}):=\arctan \frac{x_{2}}{x_{1}},
$$

and regularized in such a way that $\partial_{k} \phi(\vec{x})$ vanishes for $\vec{x} \rightarrow 0$. 
Variation of $S$ with respect to $v^{\underline{a}}(t)$ leads to the constraint

$$
\sum_{\alpha} \xi_{\alpha}^{a}=0
$$

and therefore to the vanishing of the total momentum of the $N$-particle system $[1,12]$.

The choice of gauge (2.4-5) for the dreibeins breaks asymptotically the invariance with respect to local space translations leaving, as residual symmetry [1,12], only translations local in time, and rigid rotations.

The EOM and the Gauss constraint for the $A_{\mu}$, and their solutions, are all well known (cp. [7]). We have

$$
A_{\mu}(\vec{x}, t)=-\frac{1}{2 \pi \kappa} \partial_{\mu} \sum_{\alpha} e_{\alpha} \phi\left(\vec{x}-\vec{x}_{\alpha}\right) .
$$

Let us consider now the two-body case i.e. $N=2$ in detail.

Applying the Legendre transformation to the Lagrangian (2.2) and using the relevant constraints (Gauss and (2.7)) we obtain for the two-body Hamiltonian $H$ describing relative motion

$$
H=\xi_{i} \xi_{i}
$$

where we have defined

$$
\vec{\xi}:=\frac{1}{2}\left(\vec{\xi}_{1}-\vec{\xi}_{2}\right) .
$$

Denoting the canonical particle momenta by $\vec{p}_{\alpha}$ and defining

$$
\begin{aligned}
\vec{p} & :=\frac{1}{2}\left(\vec{p}_{1}-\vec{p}_{2}\right) \\
\vec{x} & :=\frac{1}{2}\left(\vec{x}_{1}-\vec{x}_{2}\right)
\end{aligned}
$$

we obtain from $(1.2),(2.1),(2.3-5)$ and $(2.8-9)$ the relation

$$
\xi_{i}=p_{i}-\frac{\lambda}{4 \pi} \partial_{i} \phi(\vec{x})\left(H-\frac{2}{\lambda \kappa} e_{1} e_{2}\right) .
$$

Squaring it and using again (2.9) we obtain

$$
H=p^{2}-\frac{l^{2}}{r^{2}}+\frac{\bar{l}^{2}}{r^{2}}
$$

where the angular momentum for the relative motion $l(l:=\vec{x} \wedge \vec{p})$ is, according to (2.10), given by

$$
l=\bar{l}+\frac{\lambda}{4 \pi}\left(H-\frac{2}{\lambda \kappa} e_{1} e_{2}\right)
$$


with

$$
\bar{l}:=\vec{x} \wedge \vec{\xi}
$$

Note that (2.11) has the same form in case of absence of the Abelian gauge fields $[1,12]$, only the relation $(2.12)$ gets an additional term.

By applying an inverse Legendre transformation to (2.9) and using (2.10) we obtain the well known result that the coupling to the Abelian gauge fields leads only to the addition of a total time derivative to the two-particle Lagrangian

$$
L=L_{0}-\frac{e_{1} e_{2}}{2 \pi \kappa} \frac{d}{d t} \phi(\vec{x}) .
$$

Therefore the classical EOM are unchanged in comparison with $e=0$ case $[1,12]$. Due to the singular nature of $\frac{d}{d t} \phi$ this holds for noncoinciding particle positions, i.e. for $\vec{x} \neq \overrightarrow{0}$ only.

In particular, we conclude from $[1,12]$ that:

i)

$$
\dot{\xi}_{i}=0
$$

ii)

$$
\dot{x}_{i}=\frac{2 \xi_{i}}{1+\frac{\lambda \bar{l}}{2 \pi r^{2}}}
$$

leading to $\bar{l}$ being a conserved quantity and to the geometric bag formation in the case of $\lambda \bar{l}<0$ for

$$
r<r_{0}:=\left(\frac{\lambda \bar{l}}{2 \pi}\right)^{1 / 2}
$$

But, as shown in Section 4, the additional term in (2.12) leads, in the quantum case, via (2.11) to a modification of the energy levels in the confined regime.

\section{Classical Dynamics for the $D=2$ Non-Abelian Case}

We now consider the interaction of $N$ nonrelativistic particles carrying $S U(2)$-charges $Q^{a}(a=1,2,3)$ with dreibein fields $E_{\bar{\mu}} \frac{a}{\text { and }} S U(2)$-gauge fields $A_{\mu}^{a}$. The corresponding particle action $S_{\text {part }}^{(\mathrm{N})}$ is given by

$$
S_{\mathrm{part}}^{(\mathrm{N})}=S_{\mathrm{part}, 0}^{(\mathrm{N})}+\int d t \sum_{\alpha=1}^{N} Q_{\alpha}^{a}\left(A_{j, \alpha}^{a} \dot{x}_{\alpha}^{j}+A_{0, \alpha}^{a}\right)+S_{S U(2)}^{(\mathrm{N})}
$$


where $S_{S U(2)}^{(\mathrm{N})}$ is the action which is given by the symplectic form (1.7) [9]. Choosing on the sphere $S^{2}$ the spherical coordinates one gets

$$
S_{S U(2)}^{(\mathrm{N})}:=\int d t \sum_{\alpha=1}^{N} \cos \theta_{\alpha}(t) \dot{\phi}_{\alpha}(t),
$$

with $\theta, \phi$ being the angles on the sphere $S^{2}$.

The total action is now given by (see (1.1), (1.4) and (3.1-2))

$$
S^{(\mathrm{N})}=S_{0}^{(\mathrm{GR})}+S_{0}^{(\mathrm{NA})}+S_{\mathrm{part}}^{(\mathrm{N})}
$$

As again, the gauge and gravitational degrees of freedom are not coupled directly and, as in the Abelian case, the dreibeins are described by (2.3-5).

The Euler-Lagrange equation for the $S U(2)$-gauge fields $A_{\mu}^{a}$ are given by [16]:

i) the Gauss constraint

$$
F_{i j}^{a}(\vec{x}, t)=-\frac{1}{\kappa} \epsilon_{i j} \sum_{\alpha} Q_{\alpha}^{a} \delta\left(\vec{x}-\vec{x}_{a}\right),
$$

and

ii) the EOM

$$
F_{i 0}^{a}(\vec{x}, t)=\frac{1}{\kappa} \epsilon_{i j} \sum_{\alpha} Q_{\alpha}^{a} \dot{x}_{\alpha}^{j} \delta\left(\vec{x}-\vec{x}_{a}\right),
$$

where $F_{\mu \nu}^{a}$ is the $S U(2)$-field strength

$$
F_{\mu \nu}^{a}:=\partial_{\mu} A_{\nu}^{a}-\partial_{\nu} A_{\mu}^{a}+\epsilon_{a b c} A_{\mu}^{b} A_{\nu}^{c}
$$

Usually the nonlinear Gauss constraint (3.4) is solved in the axial gauge $A_{1}^{a} \equiv 0$, but then we would loose the rotational covariance.

For the derivation of the effective two-body dynamics we need only the $A_{i}^{a}(\vec{x}, t)$ at the particle positions $\vec{x}_{1,2}$. Fortunately, at these positions, the solution of (3.4) may be obtained explicitly [16] in the following form:

$$
\begin{aligned}
& A_{i, 1}^{a}=-\frac{1}{2 \pi \kappa} Q_{2}^{a} \partial_{i} \phi\left(\vec{x}_{1}-\vec{x}_{2}\right) \\
& A_{i, 2}^{a}=\frac{1}{2 \pi \kappa} Q_{1}^{a} \partial_{i} \phi\left(\vec{x}_{1}-\vec{x}_{2}\right)
\end{aligned}
$$

In order to make the arguments given in [16] rigorous we introduce a gauge field $\widetilde{A}_{i}^{a}$

$$
\widetilde{A}_{i}^{a}(\vec{x}, t):=-\frac{1}{2 \pi \kappa} \sum_{\alpha=1}^{2} Q_{\alpha}^{a} \partial_{i} \phi\left(\vec{x}-\vec{x}_{\alpha}\right),
$$

solving the linearized Gauss constraint (3.4). 
At the points $\vec{x}_{1,2}$ the potentials $\widetilde{A}_{i}^{a}$ coincide with the expressions given in $(3.7 \mathrm{a}-$ b), because the regularization of $\partial_{i} \phi$ leads to the vanishing of the self interaction terms in $(3.7 \mathrm{c})$. Furthermore the nonlinear term in the definition of $\widetilde{F}_{i j}^{a}$ (see in (3.6)) vanishes at $\vec{x}_{1,2}$, what completes the proof.

The relation (3.5) will not be discussed further as $A_{0}^{a}$ is not needed in the following.

By the same procedure as described in Section 2 we may now derive the expressions for the two-body Hamiltonian $H$ and the canonical momentum for the relative particle motion $\vec{p}$. We obtain

$$
H=\xi_{i} \xi_{i}
$$

and

$$
\xi_{i}=p_{i}-\frac{\lambda}{4 \pi} \partial_{i}(\vec{x})\left(H-\frac{2}{\lambda \kappa} Q_{1}^{a} Q_{2}^{a}\right) .
$$

Note that (3.8) and (3.9) are the same as (2.9) and (2.10) respectively with the electric charges $e_{\alpha}$ replaced by their $S U(2)$ counterparts.

Therefore, we obtain by squaring (3.9) again

$$
H=p^{2}-\frac{l^{2}}{r^{2}}+\frac{\bar{l}^{2}}{r^{2}},
$$

with

$$
l=\bar{l}+\frac{\lambda}{4 \pi}\left(H-\frac{2}{\lambda \kappa} Q_{1}^{a} Q_{2}^{a}\right),
$$

where $l$ and $\bar{l}$ are defined as before.

Finally, we have to show that $Q_{1}^{a} Q_{2}^{a}$ is a conserved quantity, i.e.

$$
\frac{d}{d t}\left(Q_{1}^{a} Q_{2}^{a}\right)=0 \text {. }
$$

In order to prove this statement we start with the non-Abelian counterpart to $(2.14)$ given by

$$
L=L_{0}-\frac{Q_{1}^{a} Q_{2}^{a}}{2 \pi \kappa} \frac{d}{d t} \phi(\vec{x})+L_{\mathrm{SU}(2)} .
$$

Then the Euler-Lagrange equations for $Q_{\alpha}^{a}$ are the Wong-equations [10,11] which take the form

$$
\dot{Q}_{1}^{a}-\frac{\dot{\phi}}{2 \pi \kappa} \epsilon_{a b c} Q_{2}^{b} Q_{1}^{c}=0
$$

By exchanging the particle indices 1 and 2 we get

$$
\dot{Q}_{2}^{a}+\frac{\dot{\phi}}{2 \pi \kappa} \epsilon_{a b c} Q_{2}^{b} Q_{1}^{c}=0
$$

Note that (3.14-15) imply that the lengths of $Q_{\alpha}$ are conserved. Thus we conclude that

$$
\dot{Q}_{1}^{a}+\dot{Q}_{2}^{a}=0
$$

which, due to (1.8), leads to the desired result (3.12). With (3.12) we conclude from (3.13) that the formulae (2.15-17) hold as in the non-Abelian case. 


\section{The Quantum-Mechanical Two-Body Problem on a Plane}

Let us start with the observation that we have, in both the Abelian and non-Abelian cases, the same structure of the classical two-body Hamiltonian

$$
H=p^{2}-\frac{l^{2}}{r^{2}}+\frac{\bar{l}^{2}}{r^{2}}
$$

with

$$
l=\bar{l}+\frac{\lambda}{4 \pi}\left(H-\frac{\Omega}{\lambda \kappa}\right)
$$

with $\Omega$, a function of the particle charges, given by

$$
\Omega=\left\{\begin{array}{ll}
2 e_{1} e_{2} & \text { Abelian case } \\
2 Q_{1}^{a} Q_{2}^{a} & \text { non-Abelian case }
\end{array} .\right.
$$

Without the gauge fields we have $\Omega=0$. Therefore, in quantizing (4.1-2) we can follow the techniques presented in $[1,12]$. However, we should keep in mind that the quantum theory requires a quantized coupling $\kappa$ in the non-Abelian case $[8,17]$

$$
4 \pi \kappa \in \mathbb{Z}
$$

Moreover, we have to properly take into account, the quantum nature of the operator $\hat{\Omega}$. We quantize the problem by considering a Schrödinger-like equation

$$
i \hbar \frac{\partial \psi(\vec{x}, t)}{\partial t}=\hat{H} \psi(\vec{x}, t)=\left[\hat{\vec{p}}^{2}-\frac{l^{2}}{r^{2}}+\frac{\bar{l}^{2}}{r^{2}}\right] \Psi(\vec{x}, t)
$$

in which the operators $\hat{H}$ and $\hat{\vec{p}}$ are defined by the usual quantization rules

$$
\hat{H}=i \hbar \frac{\partial}{\partial t}, \quad \hat{p}_{i}=\frac{\hbar}{i} \frac{\partial}{\partial x_{i}} .
$$

Note that, in the non-Abelian case, the eigenvalues of $\hat{\Omega}$ (see (1.8)) are determined by

$$
\hat{\Omega}\left|j_{12}, j_{1}, j_{2}>=\Omega\right| j_{12}, j_{1}, j_{2}>=\frac{1}{2}\left(j_{12}\left(j_{12}+1\right)-j_{1}\left(j_{1}+1\right)-j_{2}\left(j_{2}+1\right)\right) \mid j_{12}, j_{1}, j_{2}>
$$

and so the wavefunction $\Psi(\vec{x}, t)$ depends also on the eigenvalues $j_{1}, j_{2}$ and $j_{12}$. More explicitly, the wave function in (4.5), for two non-Abelian CS particles with definite quantized isospins $j_{1}$ and $j_{2}$ describes a multiplet of wave functions with $n$ components, where $n=\left|j_{1}+j_{2}\right|-\left|j_{1}-j_{2}\right|+1$. This multiplet structure and the eigenvalues of $\hat{\Omega}$ given by (4.7) will be implicitly assumed in all formulae that follow. 
For the stationary case, i.e. when $\Psi(\vec{x}, t)=\Psi_{E}(\vec{x}) e^{\frac{i E t}{\hbar}}$ we can use the angularmomentum basis and put

$$
\Psi_{E, m}=f_{E, m}(r) e^{i m \varphi}
$$

where $m$ is an integer, and find that $f_{E, m}$ satisfies a nonstandard time independent Schrödinger equation

$$
\left[-\hbar^{2}\left(\partial_{r}^{2}+\frac{1}{r} \partial_{r}-\frac{\bar{m}^{2}}{r^{2}}\right)-E\right] f_{E, m}(r)=0
$$

where, in consistency with (4.2), we have defined

$$
\hbar \bar{m}:=\hbar m-\frac{\lambda}{4 \pi}\left(E-\frac{\Omega}{\lambda \kappa}\right)
$$

i.e. $\hbar \bar{m}$ is an eigenvalue of $\bar{l}$ and $\Omega$ denotes the eigenvalue of $\hat{\Omega}$.

We see that our equation (4.9) is the same as the equation (7.6) of [12] with an important difference due to $\Omega$. The existence of $\Omega$ leads not only to the redefinition of $\bar{m}$ but, in the non-Abelian case, also to the splitting of the energy levels as for any integer or half-interger values of $j_{1}$ and $j_{2}$ there are several values of $j_{12}$ which satisfy $j_{12} \in\left(\left|j_{1}-j_{2}\right|,\left|j_{1}+j_{2}\right|\right)$. To determine the energy levels, we can, however, follow the procedure used in [12]. Thus, in particular, if we focus our attention on the interior solutions $\left(r<r_{0}\right)$ we find that they are given by

$$
f_{E, m}(r)=J_{\bar{m}}\left(\frac{\sqrt{E}}{\hbar} r\right)
$$

(restricting our attention to the more interesting case of $\lambda \bar{l}<0$ )

The energy levels are then given by the eigenvalues of $H$, which are determined by the boundary condition corresponding to the requirement that the wavefunction vanishes at $r=r_{0}$, and are given by:

$$
J_{\bar{m}}\left[\frac{\sqrt{E}}{\hbar}\left(\frac{\hbar|\lambda \bar{m}|}{2 \pi}\right)^{\frac{1}{2}}\right]=0
$$

with $\bar{m}$ given by 4.10 ).

Let us look at the case $\bar{m}>0, \lambda<0$. Then it is convenient to define

$$
\epsilon=\frac{|\lambda| E}{2 \pi \hbar}
$$

so that (4.12) takes the form

$$
J_{\bar{m}}\left(\bar{m}^{\frac{1}{2}} \epsilon^{\frac{1}{2}}\right)=0 .
$$

The Bessel function $J_{\bar{m}}$, for fixed $\bar{m}>0$, has an infinite number of positive zeroes which, in what follows, we denote by $y_{n}(\bar{m}), n=1,2$.. Thus we see that due to (4.10), the eigenvalues $\epsilon_{n}(m)$ are the positive fixed points of the equation

$$
\epsilon=f_{n}(\bar{m})=f_{n}\left(m+\frac{1}{2} \epsilon+\frac{\Omega}{4 \pi \hbar \kappa}\right)
$$


where

$$
f_{n}(\bar{m})=\frac{1}{\bar{m}} y_{n}^{2}(\bar{m})
$$

The existence of positive fixed points $\epsilon$ of (4.15) was discussed in great detail in [12], both by using various asymptotic formulae for the zeroes of Bessel's functions and also by solving (4.15) numerically. As the present case differs from the case without the gauge functions by the redefinition of $\bar{m}$, below we present the figure from [12], but this time with the interpretation that the horizontal axis denotes not $m$ but $\left(m+\frac{\Omega}{4 \pi \hbar \lambda \kappa}\right)$.

The plot looks like several curves; the lowest values correspond to the first zeroes ( ie $n=1$ ), the next ones to second zeroes ie $n=2$ etc. The points lie so close that the figure may appear as a set of lines while, in reality, we have here sets of points. The points appear to be (almost) equally spaced on each "curve" - this is due to the approximate linearity of the positions of zeros of Bessel functions as a function of $\bar{m}$. To check our values of energies we had also solved (4.15) differently; we approximated the positions of the zeroes of the Bessel functions by a linear function and solved the resultant equations for $\epsilon$. The obtained results were very similar to those of our figure thus giving us confidence in our results.

Our results show that, for each value of $m$ and so for each value of $\tilde{m}=\left(m+\frac{\Omega}{4 \pi \hbar \lambda \kappa}\right)$, there is a whole tower of values of $\epsilon$ corresponding to different zeroes of the Bessel functions. In addition, in the non-Abelian case, there is a further splitting of energy levels due to the different values of $j_{12}$ in $\Omega$. The values of $\epsilon$ increase, approximately linearly, as we take higher zeros (ie $y_{n}$ for larger $n$ ). The dependence on $m$ is only slightly more complicated; for each order of the zero there is a value of $m$ for which the energy is minimal and as we move away from this value the energy grows, approximately, linearly. As $n$ increases the minimal values of $m$ increase, again, approximately linearly.

Note that for $\bar{m}<0$ and $\lambda>0$ the corresponding energy levels are obtained by changing the sign of $m$ and $\Omega$.

We summarise our results by noting that in the interior region $r<r_{0}$, where classical solutions are only possible for a finite time interval, we can find quantum solutions which correspond to discrete bound states determined by the boundary condition at $r=r_{0}$. This shows that as in the case discussed in [12] this boundary condition defines a sort of planar geometric "bag" for the quantum state.

The discussion of the exterior solutions is again similar to what we presented in [12]. The system has no bound states and the scattering solutions are given by a superposition of Bessel functions of the first and second kind

$$
f_{E, m}(r)=A_{m}(E) J_{\bar{m}}\left(\frac{\sqrt{E}}{\hbar} r\right)+B_{m}(E) Y_{\bar{m}}\left(\frac{\sqrt{E}}{\hbar} r\right)
$$

with the ratio $\frac{A_{m}}{B_{m}}$ determined by the boundary condition of the wavefunction vanishing at $r=r_{0}$. Clearly the solutions describe a scattering on an obstruction of radius $r_{0}$, which is dynamically determined. 


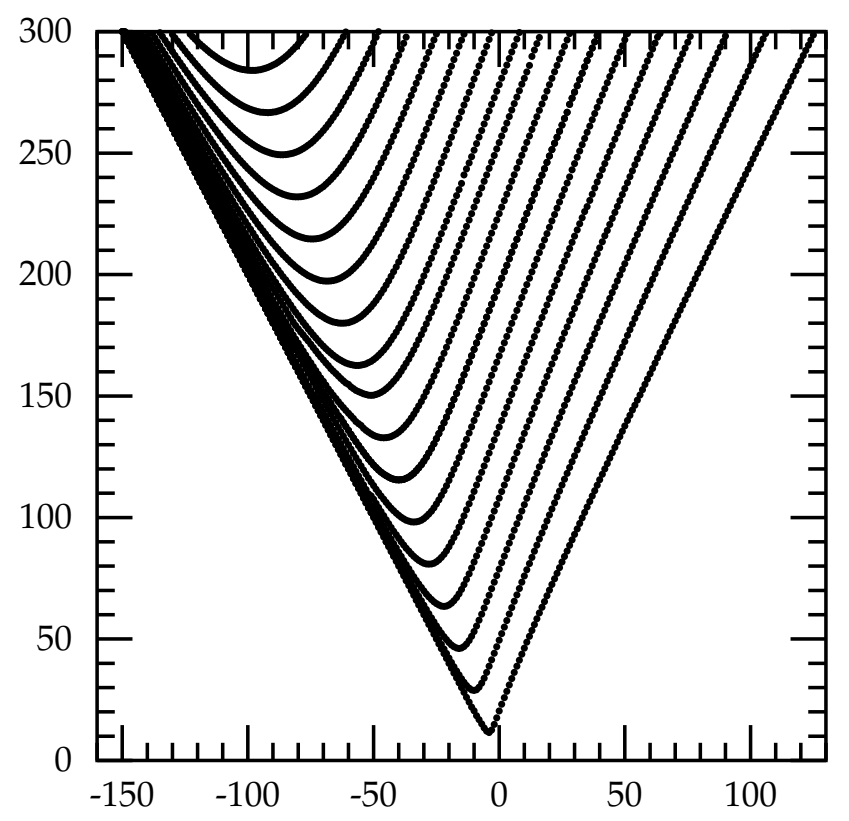

Figure 1: Energy as a function of $\tilde{m}=\left(m+\frac{\Omega}{4 \pi \hbar \lambda \kappa}\right)$

\section{$5 \quad$ Final Remarks}

In $\mathrm{D}=2+1$ dimensions one can consider four basic actions describing gravitational and gauge degrees of freedom?

- Einstein action, linear in Riemann curvature with Maxwell or Yang-Mills gauge fields

- Einstein action with (Abelian or non-Abelian) Chern-Simons gauge fields

- Translational Chern-Simons gravity action with Maxwell or Yang Mills gauge fields

- Translational Chern-Simons gravity with Chern-Simons gauge fields.

\footnotetext{
${ }^{2}$ One can consider also models with linear combinations of Maxwell and Chern-Simons terms in the gauge sector as well as both the Einstein and translational Chern-Simons terms in the gravity sector (cp.[17]). The $(2+1)$ dimensional gravity with the Einstein term supplemented by the translational Chern-Simons term was named "vector Chern-Simons gravity" in [18]
} 
In this paper we have studied the last case in this list and considered the coupling to $D=2+1$ nonrelativistic particles. We have shown that in the interacting $D=2+1$ Chern-Simons theories, with sources, the field equations take the form of field-current identities. This has allowed us to eliminate the field degrees of freedom and to obtain, without any approximation, the planar two-body interaction.

In the non-Abelian case, following [11], we have considered the motion of particles in a two-dimensional space extended by internal coordinates, in accordance with the Kaluza-Klein approach to internal symmetries.

Our basic result is a quantum-mechanical solution of the 2-body problem, describing dynamically confined particles, with the energy-dependent potential generated by the double (gravitational and gauge) Chern-Simons couplings. In our previous papers $[1,12]$ we showed that the (single) gravitational Chern-Simons coupling in $D=2$ dimensions

- leads to planar confinement

- implies the noninteger values of the quantum number $\bar{m}_{0}$

$$
\bar{m}_{0}=m-\frac{\lambda E}{4 \pi \hbar}
$$

describing the continuous values of the Abelian spin in $D=2+1$ dimensions. We see from (5.1) that the gravitational Chern-Simons coupling leads to the anyonic behaviour of massive point particles. 3

The effect of adding the Chern-Simons gauge interaction in both the Abelian and non-Abelian cases reduces to the additional shift of the continuous Abelian spin value

$$
\bar{m}_{0} \rightarrow \bar{m}=\bar{m}_{0}+\frac{\Omega}{4 \pi \kappa \hbar} .
$$

Thus we see that in the Abelian case we have anyonic values of the angular momentum, shifted by a term proportional to the product of Abelian gauge charges. In the non-Abelian case the shift is given by the eigenvalues of the operator $\frac{\hat{\Omega}}{2}(1.8)$, described explicitly by (4.7) $i e$ it is matrix valued. We see from our results that the planar confinement remains valid as in the purely gravitational case. In the nonAbelian case we have a new effect - the splitting of energy levels.

\section{Acknowledgment}

The work reported in this paper was completed when two of us (JL and WJZ) were visiting, respectively, the University of Valencia, Spain and MIT, USA. They would like to thank their hosts for their hospitality and for financial support making their visits possible, respectively, JL - Generalitat Valenciana and WJZ - Center for Theoretical Physics, MIT.

\footnotetext{
${ }^{3}$ Such an observation was made earlier in [19]
} 


\section{References}

[1] J. Lukierski, P.C. Stichel, W.J. Zakrzewski, Phys. Lett. B484, 315 (2000).

[2] M. Stone "Quantum Hall Effect", World Scientific, Singapore, 1992.

[3] G. Burns, High Temperature Superconductivity, Academic Press, 1992.

[4] R. Iengo and K. Lechner, Phys. Rep. 213, 179 (1992).

[5] J. Fröhlich, in "Nonperturbative Quantum Field Theory", Ed. G.t'Hooft, Plenum Press, New York, 1998.

[6] A.P. Balachandran, M. Bordeau, S. Io, Int. Journ. Mod. Phys. A5, 2423 (1990).

[7] R. Jackiw, S.Y. Pi, Phys. Rev. D42, 3500 (1990); R. Jackiw, Ann. Phys. (NY), 201, 83 (1990).

[8] D. Bak, P. Jackiw and So-Young Pi, Phys. Rev. D49, 6778 (1994).

[9] A. Alekseev, L. Faddeev, S. Shatashvili, Journ. Geom. Phys. 5, 391 (1989).

[10] S.K. Wong, Nuovo Cim. 65A, 689 (1970).

[11] R. Kerner, Ann. Inst. H. Poincaré, 9, 141 (1968).

[12] J. Lukierski, P.C. Stichel, W.J. Zakrzewski, "From Gauging Nonrelativistic Translations to N-Body Dynamics", Ann. Phys. (NY), in press, preprint hep th/0007102.

[13] P.C. Stichel, Phys. Lett. B456, 129 (1998).

[14] P.C. Stichel, Ann. Phys. (NY), 285, 161 (2000).

[15] P.C. Stichel and W.J. Zakrzewski, in preparation.

[16] T. Lee, Ph. Oh, Ann. Phys. 235, 413 (1994).

[17] S. Deser, R. Jackiw and S. Templeton, Ann. Phys. (NY), 140, 372 (1982).

[18] C. Aragone, P.J. Arias and A. Khoudeir, Nuovo Cim. B109, 303 (1994).

[19] C. Aragone, P.J. Arias, "More Gravitational Anyons", hep-th/9309131; Y.M. Cho, D.H. Park and C.G. Han, Phys. Rev. D 43, 1421 (1991) 